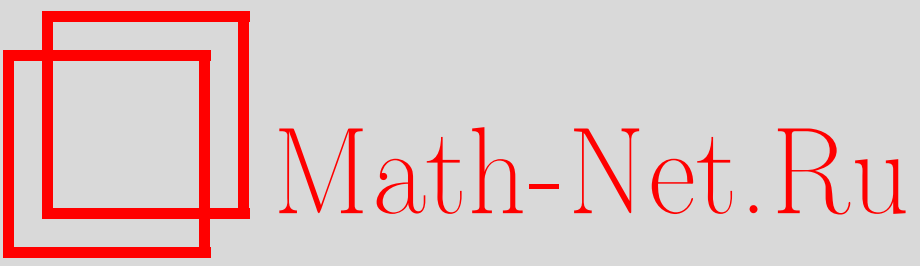

О. О. Белова, Редукция расслоений грассманоподобного многообразия центрированных плоскостей при нормализации, Итоги науки и техн. Сер. Соврем. мат. и ее прил. Темат. обз., 2020, том 180, 3-8

DOI: https://doi.org/10.36535/0233-6723-2020-180-3-8

Использование Общероссийского математического портала Math-Net.Ru подразумевает, что вы прочитали и согласны с пользовательским соглашением

http: //www. mathnet.ru/rus/agreement

Параметры загрузки:

IP: 54.224 .60 .19

26 апреля 2023 г., 17:04:42 


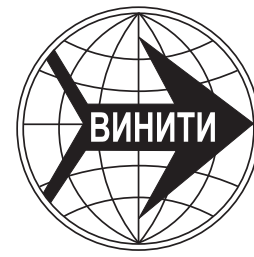

ИТОГИ НАУКИ И ТЕХНИКИ.

Современная математика и ее приложения.

Тематические обзоры.

Том 180 (2020). C. 3-8

DOI: $10.36535 / 0233-6723-2020-180-3-8$

УДК 514.76

\title{
РЕДУКЦИЯ РАССЛОЕНИЙ \\ ГРАССМАНОПОДОБНОГО МНОГООБРАЗИЯ \\ ЦЕНТРИРОВАННЫХ ПЛОСКОСТЕЙ ПРИ НОРМАЛИЗАЦИИ
}

(c) 2020 г. О. О. БЕЛОВА

\begin{abstract}
АннотАция. В проективном пространстве $P_{n}$ рассмотрено грассманоподобное многообразие $G r^{*}(m, n)$ центрированных плоскостей. С ним ассоциировано главное расслоение. Произведены нормализация многообразия $G r^{*}(m, n)$ полями нормалей первого и второго порядков и адаптация репера соответствующим нормалям. Исследованы полунормализованные пространства первого и второго рода, а также нормализованное пространство. Установлена динамика изменений соответствующих расслоений при переходе от пространства $G r^{*}(m, n)$ к нормализованному пространству.
\end{abstract}

Ключевые слова: метод Картана-Лаптева, грассманоподобное многообразие центрированных плоскостей, нормализация, редукция.

\section{REDUCTION OF BUNDLES OF A GRASSMANN-LIKE MANIFOLD OF CENTERED PLANES UNDER NORMALIZATION}

\author{
(c) 2020 O. O. BELOVA
}

\begin{abstract}
In the projective space $P_{n}$, we consider the Grassmann-like manifold $G r^{*}(m, n)$ of centered planes and the associated principal bundle. We perform the normalization of the manifold $G r^{*}(m, n)$ by the normal fields of the first and second orders and the the adaptation of the bundle corresponding to these normals. Semi-normalized spaces of the first and second kind and the normalized space are examined. The dynamics of changes of the corresponding bundles is established when passing from the space $G r^{*}(m, n)$ to the normalized space.
\end{abstract}

Keywords and phrases: Cartan-Laptev method, Grassmann-like manifold of centered planes, normalization, reduction.

AMS Subject Classification: 53A20, 53A99

1. Введение. В работе используются метод Картана-Лаптева, базирующийся на теории конечных и бесконечных групп Ли, исчислении внешних форм Э. Картана и теории Г. Ф. Лаптева полей геометрических объектов (см. [12]). При использовании метода Картана подвижного репера исследование геометрии самого многообразия с фиксированной на нем геометрической структурой сводится к исследованию геометрии другого многообразия: пространства расслоения реперов над данным многообразием или подрасслоений этого расслоения. Таким образом, автоматически строится аналитический аппарат, который наиболее приспособлен к исследованию исходной структуры (см. [6]). Метод продолжений и охватов Лаптева основан на инвариантном дифференциально-алгебраическом аппарате структурных дифференциальных форм рассматриваемых расслоений (см. [5, 13]). 
Поскольку поверхность $X_{m}$ можно рассматривать как $m$-мерное многообразие $m$-мерных центрированных плоскостей $T_{m}$ (см. [8]), то при исследовании грассманоподобного многообразия центрированных плоскостей используется аналог нормализации А. П. Нордена (см. [4, 11]), который осуществлял нормализацию поверхности полями нормалей первого и второго рода и обозначал их соответственно через $P_{I}$ и $P_{I I}$. В [10] Ю. Г. Лумисте ввел аналогичную нормализацию многообразия $m$-мерных плоскостей в проективном пространстве.

В данной статье проводится анализ динамики изменений расслоений при последовательных канонизациях и переходе от многообразия $G r^{*}(m, n)$ к нормализованному многообразию.

2. Грассманоподобное многообразие центрированных плоскостей. Отнесем $n$-мерное проективное пространство $P_{n}$ к подвижному реперу $\left\{A, A_{I}\right\}, I, \ldots=1, \ldots, n$, инфинитезимальные перемещения которого определяются формулами

$$
d A=\theta A+\omega^{I} A_{I}, \quad d A_{I}=\theta A_{I}+\omega_{I}^{J} A_{J}+\omega_{I} A,
$$

где 1-форма $\theta$ играет роль множителя пропорциональности, а формы Пфаффа $\omega^{I}, \omega_{J}^{I}, \omega_{I}$ удовлетворяют структурным уравнениям Картана

$$
\begin{aligned}
& D \omega^{I}=\omega^{J} \wedge \omega_{J}^{I}, \\
& D \omega_{J}^{I}=\omega_{J}^{K} \wedge \omega_{K}^{I}+\delta_{J}^{I} \omega_{K} \wedge \omega^{K} \omega_{J} \wedge \omega^{I}, \\
& D \omega_{I}=\omega_{I}^{J} \wedge \omega_{J}
\end{aligned}
$$

проективной группы $G P(n)$, действующей эффективно в пространстве $P_{n}$.

Замечание 1. Проективное пространство $P_{n}$ можно представить как фактор-пространство $L_{n+1} / \sim$ линейного пространства $L_{n+1}$ по отношению эквивалентности (коллинеарности) $\sim$ ненулевых векторов (см. [15]). Таким образом, аналитическая точка $\bar{A} \in L_{n+1} \backslash\{\overline{0}\}$ используется в формулах, но черточка опускается для упрощения записи, а геометрическая точка $A=\{\lambda \bar{A}\} \in P_{n}$, $\lambda \in R \backslash 0,-$ в рассуждениях. Уравнения (1) описывают инфинитезимальный закон перемещения сопутствующего репера из проективного слоя вдоль рассматриваемого многообразия. Во многих геометрических исследованиях (см. [17]) и в работах, где применяются математические методы для решения физических задач (см., например, $[1,7]$ ), используются уравнения аналогичных инфинитезимальных перемещений реперов. Первые слагаемые в (1) не существенны, так как коллинеарным аналитическим точкам соответствует одна геометрическая точка, т.е. $\theta$ не влияет на смещение $d A$. Формы Пфаффа $\omega^{I}, \omega_{J}^{I}, \omega_{I}$ (1-формы) содержат параметры, от которых зависит семейство реперов, и их дифференциалы: $\omega=\omega(u, d u)$ (см. [18]).

Замечание 2. В данной работе применяется неоднородный аналитический аппарат с деривационными формулами (1) и структурными уравнениями (2) (см. [15]). Данный аппарат наиболее удобен при выделении подгрупп проективной группы.

Определение 1. Грассманоподобным многообразием $G r^{*}(m, n)$ чентрированных плоскостей называется многообразие $m$-мерных плоскостей $n$-мерного проективного пространства $P_{n}$, проходящих через фиксированную точку (см. [3]).

Многообразие $G r^{*}(m, n)$ задается уравнениями

$$
\omega^{a}=\Lambda_{\alpha}^{a} \omega^{\alpha}+\Lambda_{\alpha}^{a b} \omega_{b}^{\alpha},
$$

где $\Lambda_{\alpha}^{a}, \Lambda_{\alpha}^{a b}$ - произвольные функции, являющиеся компонентами фундаментального объекта (см. [8]). Здесь и в дальнейшем индексы принимают следующие значения: $a, \ldots=1, \ldots, m$; $\alpha, \ldots=m+1, \ldots, n$.

Уравнения (3) получаются при специализации подвижного репера (вершины $A, A_{a}$-на плоскости $P_{m}$, а центр $A$ фиксирован). При этом формы $\omega^{\alpha}, \omega_{a}^{\alpha}$, обнуление которых фиксирует центрированную плоскость $P_{m}^{0} \omega^{a}$, являются главными, формы $\omega^{\alpha}, \omega_{a}^{\alpha}$ - базисные, а остальные формы слоевые.

Замечание 3. Если $G r(m, n)$ - многообразие Грассмана (см. [2]), то

$$
\operatorname{dim} G r^{*}(m, n)=\operatorname{dim} G r(m, n)=(n-m)(m+1) .
$$


Фундаментальный объект $\Lambda=\left\{\Lambda_{\alpha}^{a}, \Lambda_{\alpha}^{a b}\right\}$ является квазитензором, так как его компоненты удовлетворяют дифференциальным сравнениям по модулю базисных форм $\omega^{\alpha}, \omega_{a}^{\alpha}$ :

$$
\Delta \Lambda_{\alpha}^{a}+\Lambda_{\alpha}^{a b} \omega_{b}+\omega_{\alpha}^{a} \equiv 0, \quad \Delta \Lambda_{\alpha}^{a b} \equiv 0,
$$

причем дифференциальный тензорный оператор $\Delta$ действует по закону

$$
\Delta \Lambda_{\alpha}^{a b}=d \Lambda_{\alpha}^{a b}+\Lambda_{\alpha}^{c b} \omega_{c}^{a}+\Lambda_{\alpha}^{a c} \omega_{c}^{b}-\Lambda_{\beta}^{a b} \omega_{\alpha}^{\beta} .
$$

Замечание 4. Фундаментальный объект $\Lambda$ содержит подтензор $\Lambda_{\alpha}^{a b}$, обращение в нуль которого характеризует такое подмногообразие, когда центр $A$ описывает $(n-m)$-поверхность с уравнениями $\omega^{a}=\Lambda_{\alpha}^{a} \omega^{\alpha}$.

3. Главное расслоение. Специализация подвижного репера для грассманоподобного многообразия $G r^{*}(m, n)$ центрированных плоскостей приводит к главному расслоению $G\left(G r^{*}\right)$, типовым слоем которого является подгруппа $G$ стационарности центрированной плоскости $P_{m}^{0}$, а базой-многообразие $G r^{*}(m, n)$, причем $\operatorname{dim} G=n^{2}-m n+m^{2}+n$. Пространством расслоения $G\left(G r^{*}\right)$ (см. [6]) является проективная группа $G P(n)$, а проекция $\pi: G P(n) \rightarrow G r^{*}(m, n)$ относит произвольному элементу группы $G P(n)$ ту плоскость $P_{m}^{0}$ многообразия $G r^{*}(m, n)$, которая инвариантна под действием этого элемента.

Базисные формы $\omega^{\alpha}, \omega_{a}^{\alpha}$ удовлетворяют структурным уравнениям Картана $(2)$, поэтому получим

$$
\begin{aligned}
& D \omega^{\alpha}=-\omega_{\beta}^{\alpha} \wedge \omega^{\beta}+\Lambda_{\beta}^{a} \omega^{\beta} \wedge \omega_{a}^{\alpha}+\Lambda_{\beta}^{a b} \omega_{b}^{\beta} \wedge \omega_{a}^{\alpha}, \\
& D \omega_{a}^{\alpha}=\left(\delta_{\beta}^{\alpha} \omega_{a}^{b}-\delta_{a}^{b} \omega_{\beta}^{\alpha}\right) \wedge \omega_{b}^{\beta}+\omega_{a} \wedge \omega^{\alpha} .
\end{aligned}
$$

Внешние дифференциалы слоевых форм имеют вид

$$
\begin{gathered}
D \omega_{b}^{a}=\omega_{b}^{c} \wedge \omega_{c}^{a}+\omega_{b \alpha}^{a} \wedge \omega^{\alpha}+\omega_{b \alpha}^{a c} \wedge \omega_{c}^{\alpha} ; \\
D \omega_{a}=\omega_{a}^{b} \wedge \omega_{b}-\omega_{\alpha} \wedge \omega_{a}^{\alpha} ; \\
D \omega_{\beta}^{\alpha}=\omega_{\beta}^{\gamma} \wedge \omega_{\gamma}^{\alpha}+\omega_{\beta \gamma}^{\alpha} \wedge \omega^{\gamma}+\omega_{\beta \gamma}^{\alpha a} \wedge \omega_{a}^{\gamma} ; \\
D \omega_{\alpha}^{a}=\omega_{\alpha}^{b} \wedge \omega_{b}^{a}+\omega_{\alpha}^{\beta} \wedge \omega_{\beta}^{a}+\omega_{\alpha \beta}^{a} \wedge \omega^{\beta}+\omega_{\alpha \beta}^{a b} \wedge \omega_{b}^{\beta} ; \\
D \omega_{\alpha}=\omega_{\alpha}^{a} \wedge \omega_{a}+\omega_{\alpha}^{\beta} \wedge \omega_{\beta},
\end{gathered}
$$

где

$$
\begin{gathered}
\omega_{b \alpha}^{a}=\delta_{b}^{a} \Lambda_{\alpha}^{c} \omega_{c}+\delta_{b}^{a} \omega_{\alpha}+\Lambda_{\alpha}^{a} \omega_{b}, \quad \omega_{b \alpha}^{a c}=\delta_{b}^{a} \lambda_{\alpha}^{e c} \omega_{e}-\delta_{b}^{c} \omega_{\alpha}^{a}+\Lambda_{\alpha}^{a c} \omega_{b}, \\
\omega_{\beta \gamma}^{\alpha}=\delta_{\beta}^{\alpha} \Lambda_{\gamma}^{a} \omega_{a}+\delta_{\beta}^{\alpha} \omega_{\gamma}+\delta_{\gamma}^{\alpha} \omega_{\beta}, \quad \omega_{\beta \gamma}^{\alpha a}=\delta_{\beta}^{\alpha} \Lambda_{\gamma}^{b a} \omega_{b}+\delta_{\gamma}^{a} \omega_{\beta}^{a}, \\
\omega_{\alpha \beta}^{a}=\Lambda_{\beta}^{a} \omega_{\alpha}, \quad \omega_{\alpha \beta}^{a b}=\Lambda_{\beta}^{a b} \omega_{\alpha} .
\end{gathered}
$$

Теорема 1. Главное расслоение $G\left(G r^{*}\right)$ многообразия $G r^{*}(m, n)$ содержит следующие пять фактор-расслоений:

1. фактор-расслоение линейных реперов $L_{m^{2}}\left(P_{n}\right)$ со структурными уравнениями (4), (5) и слоевыми формами $\omega_{b}^{a}$. Данные реперы принадлежат плоскостлм $P_{m}^{0}$. Типовым слоем данного расслоения является линейная фактор-группа $L_{m^{2}}=G L(m) \subset G$, которая действует в пучке прямых, принадлежащих образующей плоскости $P_{m}^{0}$;

2. фактор-расслоение нормальных линейных реперов $\mathfrak{L}_{(n-m)^{2}}\left(P_{n}\right)$ со структурными уравнениями (4), (7) и слоевыми формами $\omega_{\beta}^{\alpha}$. Данное расслоение является двойственным фактор-расслоению линейных реперов. Типовым слоем здесь является линейная фактор-группа $\mathfrak{L}_{(n-m)^{2}}=$ $G L(n-m) \subset G$, действующал в проективном фактор-пространстве $P_{n-m-1}=P_{n} / P_{m}^{0}$;

3. фактор-расслоение плоскостных коаффинных реперов $C_{m(m+1)}\left(P_{n}\right)$ со структурными уравнениями (4)-(6) и слоевыми формами $\omega_{b}^{a}, \omega_{a}$. Данные реперы принадлежат плоскости $P_{m}^{0}$. Типовым слоем указанного расслоения является коаффинная (чентропроективная) факторгруппа $C_{m(m+1)}=G A^{*}(m) \subset G$, действующая в плоскости $P_{m}^{0}$; 
4. аффинное фактор-расслоение $H_{k}\left(P_{n}\right), k=n(n-m)+m^{2}$, со структурными уравнениями (4), (5), (7), (8) и слоевыми формами $\omega_{b}^{a}, \omega_{\beta}^{\alpha} u \omega_{\alpha}^{a}$. Типовым слоем здесь будет афффиннал факторгруппа (см. [16]) $H_{k}$ группы $G \subset G P(n)$, действующая в пучке прямых с иентром в точке $A$;

5. максимальное фактор-расслоение, составленное из фактор-расслоения плоскостных коафбинных реперов и афбинного фактор-расслоения, со структурными уравнениями (4)-(8) и слоевыми формами $\omega_{b}^{a}, \omega_{a}, \omega_{\beta}^{\alpha} u \omega_{\alpha}^{a}$.

Замечание 5. Подгруппы группы $G$ связаны отношением

$$
C_{m(m+1)} \cap H_{k}=L_{m^{2}} .
$$

Произведем нормализацию многообразия $G r^{*}(m, n)$ полями следующих геометрических образов: нормалью первого рода (см. [9]), т.е. $(n-m)$-мерной плоскостью $N_{n-m}$, пересекающейся с плоскостью $P_{m}^{0}$ в точке $A$, и нормалью второго рода (см. [9]), т.е. $(m-1)$-мерной плоскостью $N_{m-1}$, принадлежащей плоскости $P_{m}^{0}$ и не проходящей через ее центр $A$.

Проследим динамику изменений расслоения $G\left(G r^{*}\right)$ при последовательных канонизациях:

(1) первая канонизация: репер адаптирован нормали первого рода $N_{n-m}$, т.е. вершины $A_{\alpha}$ помещены на плоскость $N_{n-m}$. Полученное пространство будем называть полунормализованным пространством первого рода и обозначать $G r^{* 1}$;

(2) вторая канонизаиия: репер адаптирован нормали второго рода $N_{m-1}$, т.е. вершины $A_{a}$ помещены на плоскость $N_{m-1}$. Полученное пространство будем называть полунормализованным пространством второго рода и обозначать $G r^{* 2}$;

(3) полная канонизация: репер адаптирован нормалям первого и второго рода, т.е. одновременное размещение данных вершин в соответствующих нормалях. Полученное пространство будем называть нормализованным пространством и обозначать $G r^{* 1,2}$.

3.1. Расслоение $G^{1}\left(G r^{*}\right)$. Поместим вершины $A_{\alpha}$ в нормаль первого рода $N_{n-m}$; тогда

$$
\omega_{\alpha}^{a}=g_{\alpha \beta}^{a} \omega^{\beta}+g_{\alpha \beta}^{a b} \omega_{b}^{\beta},
$$

т.е. слоевые формы $\omega_{\alpha}^{a}$ становятся главными, причем имеют место дифференциальные сравнения

$$
\Delta g_{\alpha \beta}^{a}+g_{\alpha \beta}^{a b} \omega_{b}-\Lambda_{\beta}^{a} \omega_{\alpha} \equiv 0, \quad \Delta g_{\alpha \beta}^{a b}-\Lambda_{\beta}^{a b} \omega_{\alpha} \equiv 0 .
$$

Из структурных уравнений (2) находим

$$
\begin{gathered}
D \omega_{b}^{a}=\omega_{b}^{c} \wedge \omega_{c}^{a}+\omega_{b \alpha}^{a} \wedge \omega^{\alpha}+\left(\Lambda_{\alpha}^{a c} \omega_{b}+g_{\beta \alpha}^{a c} \omega_{b}^{\beta}+\delta_{b}^{a} \Lambda_{\beta}^{e c} \omega_{e}\right) \wedge \omega_{c}^{\alpha}+g_{\alpha \beta}^{a} \omega_{b}^{\alpha} \wedge \omega^{\beta} ; \\
D \omega_{\beta}^{\alpha}=\omega_{\beta}^{\gamma} \wedge \omega_{\gamma}^{\alpha}+\omega_{\beta \gamma}^{\alpha} \wedge \omega^{\gamma}+\delta_{\beta}^{\alpha} \Lambda_{\gamma}^{a b} \omega_{a} \wedge \omega_{b}^{\gamma}+g_{\beta \gamma}^{a} \omega^{\gamma} \wedge \omega_{a}^{\alpha}+g_{\beta \gamma}^{a b} \omega_{b}^{\gamma} \wedge \omega_{a}^{\alpha} ; \\
D \omega_{a}=\omega_{a}^{b} \wedge \omega_{b}-\omega_{\alpha} \wedge \omega_{a}^{\alpha} \\
D \omega_{\alpha}=\omega_{\alpha}^{\beta} \wedge \omega_{\beta}-g_{\alpha \beta}^{a} \omega_{a} \wedge \omega^{\beta}-g_{\alpha \beta}^{a b} \omega_{a} \wedge \omega_{b}^{\beta} .
\end{gathered}
$$

Отсюда вытекает следующая теорема.

Теорема 2. Главное расслоение $G\left(G r^{*}\right)$ при первой канонизации сужается до главного расслоения $G^{1}\left(G r^{*}\right)$ с типовым слоем - подгруппой стационарности $G^{1} \subset G$ пары аффинно-дополнительных плоскостей $\left\{P_{m}, N_{n-m}\right\}$. В подрасслоении $G^{1}\left(G r^{*}\right)$ выделяются следуюшие четыре фактор-расслоения:

(1) расслоение плоскостных линейных реперов со структурными уравнениями (4), (10);

(2) расслоение нормалъных линейных реперов (4), (11);

(3) расслоение плоскостных коаффинных реперов (4), (10), (12);

(4) расслоение нормальных коаффинных реперов (4), (11), (13).

Замечание 6. Указанные реперы рассматриваются на полунормализованном многообразии $G r^{* 1}$. 
3.2. Расслоение $G^{2}\left(G r^{*}\right)$. Если отказаться от предыдущей канонизации и поместить вершины $A_{a}$ в нормаль второго рода $N_{m-1}$, то

$$
\omega_{a}=g_{a \alpha} \omega^{\alpha}+g_{a \alpha}^{b} \omega_{b}^{\alpha},
$$

т.е. слоевые формы $\omega_{a}$ становятся главными, причем

$$
\Delta g_{a \alpha} \equiv 0, \quad \Delta g_{a \alpha}^{b}+\delta_{a}^{b} \omega_{\alpha} \equiv 0 .
$$

Из структурных уравнений (2) находим

$$
\begin{gathered}
D \omega_{b}^{a}=\omega_{b}^{c} \wedge \omega_{c}^{a}+\delta_{b}^{a} \omega_{\alpha} \wedge \omega^{\alpha}-\delta_{b}^{c} \omega_{\alpha}^{a} \wedge \omega_{c}^{\alpha}+\left(\delta_{b}^{a} g_{c \alpha} \Lambda_{\beta}^{c}+g_{b \alpha} \Lambda_{\beta}^{a}\right) \omega^{\alpha} \wedge \omega^{\beta}+ \\
+\left(\delta_{b}^{a} g_{e \alpha} \Lambda_{\beta}^{e c}-\delta_{b}^{a} g_{e \beta}^{c} \Lambda_{\alpha}^{e}+g_{b \alpha} \Lambda_{\beta}^{a c}-g_{b \beta}^{c} \Lambda_{\alpha}^{a}\right) \omega^{\alpha} \wedge \omega_{c}^{\beta}+\left(g_{b \alpha}^{c} \Lambda_{\beta}^{a e}+\delta_{b}^{a} g_{d \alpha}^{c} \Lambda_{\beta}^{d e}\right) \omega_{c}^{\alpha} \wedge \omega_{e}^{\beta} \\
D \omega_{\beta}^{\alpha}=\omega_{\beta}^{\gamma} \wedge \omega_{\gamma}^{\alpha}+\left(\delta_{\beta}^{\alpha} \omega_{\gamma}+\delta_{\gamma}^{\alpha} \omega_{\beta}\right) \wedge \omega^{\gamma}+\omega_{\beta}^{a} \wedge \omega_{a}^{\alpha}+\delta_{\beta}^{\alpha} g_{a \gamma} \Lambda_{\mu}^{a} \omega^{\gamma} \wedge \omega^{\mu}+ \\
+\left(\delta_{\beta}^{\alpha} g_{a \gamma} \Lambda_{\mu}^{a b}-\delta_{\beta}^{\alpha} g_{a \mu}^{b} \Lambda_{\gamma}^{a}\right) \omega^{\gamma} \wedge \omega_{b}^{\mu}+\delta_{\beta}^{\alpha} g_{a \gamma}^{b} \Lambda_{\mu}^{a c} \omega_{b}^{\gamma} \wedge \omega_{c}^{\mu} \\
D \omega_{\alpha}^{a}=\omega_{\alpha}^{b} \wedge \omega_{b}^{a}+\omega_{\alpha}^{\beta} \wedge \omega_{\beta}^{a}+\Lambda_{\beta}^{a} \omega_{\alpha} \wedge \omega^{\beta}+\Lambda_{\beta}^{a b} \omega_{\alpha} \wedge \omega_{b}^{\beta} \\
D \omega_{\alpha}=\omega_{\alpha}^{\beta} \wedge \omega_{\beta}+g_{a \beta} \omega_{\alpha}^{a} \wedge \omega^{\beta}+g_{a \beta}^{b} \omega_{\alpha}^{a} \wedge \omega_{b}^{\beta} .
\end{gathered}
$$

Таким образом, справедлива следующая теорема.

Теорема 3. При второй канонизации главное расслоение $G\left(G r^{*}\right)$ сужсается до главного расслоения $G^{2}\left(G r^{*}\right)$ с типовым слоем-подгруппой стационарности $G^{2} \subset G$ пары $\left\{A, N_{m-1}\right\}$. B подрасслоении $G^{2}\left(G r^{*}\right)$ выделяются четыре фактор-расслоения:

(1) расслоение плоскостных линейных реперов (4), (15);

(2) расслоение нормалъных линейных реперов (4), (16);

(3) расслоение $H\left(G r^{*}\right)(4)$, (15)-(17) с типовым слоем $H$-аффинной фактор-группой (см. [15]);

(4) расслоение нормальных коаффинных реперов (4), (16), (18).

Замечание 7. Указанные реперы рассматриваются на полунормализованном многообразии $G r^{* 2}$.

3.3. Расслоение $G^{1,2}\left(G r^{*}\right)$. Произведем одновременно канонизации, рассмотренные в предыдущих пунктах, т.е. $A_{\alpha} \in N_{n-m}, A_{a} \in N_{m-1}$. При этом будут выполняться одновременно условия (9) и (14). В этом случае структурные уравнения (2) примут вид

$$
\begin{aligned}
& D \omega_{b}^{a}=\omega_{b}^{c} \wedge \omega_{c}^{a}+ \delta_{b}^{a} \omega_{\alpha} \wedge \omega^{\alpha}+\left(\delta_{b}^{a} g_{c \alpha} \Lambda_{\beta}^{c}+g_{b \alpha} \Lambda_{\beta}^{a}\right) \omega^{\alpha} \wedge \omega^{\beta}+ \\
&+\left(\delta_{b}^{a} g_{e \alpha} \Lambda_{\beta}^{e c}-\delta_{b}^{a} g_{e \beta}^{c} \Lambda_{\alpha}^{e}+g_{b \alpha} \Lambda_{\beta}^{a c}-\delta_{b}^{c} g_{\beta \alpha}^{a}-g_{b \beta}^{c} \Lambda_{\alpha}^{a}\right) \omega^{\alpha} \wedge \omega_{c}^{\beta}+ \\
&+\left(g_{b \alpha}^{c} \Lambda_{\beta}^{a e}+\delta_{b}^{c} g_{\alpha \beta}^{a e}+\delta_{b}^{a} g_{d \alpha}^{c} \Lambda_{\beta}^{d e}\right) \omega_{c}^{\alpha} \wedge \omega_{e}^{\beta} \\
& D \omega_{\beta}^{\alpha}=\omega_{\beta}^{\gamma} \wedge \omega_{\gamma}^{\alpha}+\left(\delta_{\gamma}^{\alpha} \omega_{\beta}+\delta_{\beta}^{\alpha} \omega_{\gamma}\right) \wedge \omega^{\gamma}+\delta_{\beta}^{\alpha} g_{a \gamma} \Lambda_{\mu}^{a} \omega^{\gamma} \wedge \omega^{\mu}+ \\
&+\left(\delta_{\mu}^{\alpha} g_{\beta \gamma}^{a}+\delta_{\beta}^{\alpha} g_{b \gamma} \Lambda_{\mu}^{b a}-\delta_{\beta}^{\alpha} g_{b \mu}^{a} \Lambda_{\gamma}^{a}\right) \omega^{\gamma} \wedge \omega_{a}^{\mu}+\left(\delta_{\mu}^{\alpha} g_{\beta \gamma}^{b a}+\delta_{\beta}^{\alpha} g_{c \gamma}^{a} \Lambda_{\mu}^{c b}\right) \omega_{a}^{\gamma} \wedge \omega_{b}^{\mu} \\
& D \omega_{\alpha}=\omega_{\alpha}^{\beta} \wedge \omega_{\beta}+g_{\alpha \beta}^{a} g_{a \gamma} \omega^{\beta} \wedge \omega^{\gamma}+\left(g_{\alpha \beta}^{b} g_{b \gamma}^{a}-g_{\alpha \gamma}^{b a} g_{b \beta}\right) \omega^{\beta} \wedge \omega_{a}^{\gamma}+g_{\alpha \beta}^{a b} g_{a \gamma}^{c} \omega_{b}^{\beta} \wedge \omega_{c}^{\gamma}
\end{aligned}
$$

Итак, верна следующая теорема.

Теорема 4. При одновременной канонизации из сужения $G^{1,2}\left(G r^{*}\right)$ главного расслоения $G\left(G r^{*}\right)$, где $G^{1,2}-$ подгруппа стачионарности чентрированной $(n-m)$-пары $\left\{N_{n-m}^{*}, N_{m-1}\right\}$ (см. [14]), выделяются следующие три фактор-расслоения:

(1) расслоение плоскостных линейных реперов (4), (19);

(2) расслоение нормальных линейных реперов (4), (20);

(3) расслоение нормальных коаффинных реперов (4), (20), (21). 
Замечание 8. Все реперы, обозначенные в теореме 4, рассматриваются на нормализованном многообразии $G r^{* 1,2}$.

\section{СПИСОК ЛИТЕРАТУРЫ}

1. Аль-Хассани M. А., Молдованова E. А. Отображение аффинного пространства в многообразие нульпар проективного пространства// Изв. Томск. пед. ун-та. - 2013. - 322, № 2. - С. 24-28.

2. Белова О. О. Связности в расслоениях, ассоциированных с многообразием Грассмана и пространством центрированных плоскостей// Фундам. прикл. мат. - 2008. - 14, № 2. - С. 29-67.

3. Белова О. О. Грассманоподобное многообразие центрированных плоскостей// Мат. заметки. - 2018. - 104, № 6. - C. 812-822.

4. Евтушик Л. Е. Структуры высших порядков. - М.: Интеллект-центр, 2014.

5. Евтушик Л. Е., Лумисте Ю. Г., Остиану Н. М., Широков А. П. Дифференциально-геометрические структуры на многообразиях// Итоги науки и техн. Сер. Пробл. геом. - 1979. - 9. - С. 5-246.

6. Кириченко В. Ф. Дифференциально-геометрические структуры на многообразиях. - М.: МПГУ, 2003.

7. Кривоносов Л. Н., Лукъянов В. А. Уравнения Эйнштейна на четырехмерном многообразии конформной связности без кручения// Ж. Сиб. фед. ун-та. Сер. мат. физ. - 2012. - 5, № 3. - С. 393-408.

8. Лаптев $Г$. Ф. Дифференциальная геометрия погруженных многообразий. Теоретико-групповой метод дифференциально-геометрических исследований// Тр. Моск. мат. о-ва. - 1953. - 2. - С. 275-382.

9. Лаптев $Г$. Ф., Остиану Н. М. Распределения $m$-мерных линейных элементов в пространстве проективной связности// Тр. геом. сем. ВИНИТИ. - 1971. - 3. - С. 49-94.

10. Лумисте Ю. Г. Индуцированные связности в погруженных проективных и аффинных расслоениях// Уч. зап. Тартуск. ун-та. - 1965. - 177. - С. 6-41.

11. Норден А. П. Пространства аффинной связности. - М.: Наука, 1976.

12. Остиану H. M. Метод Картана-Лаптева в исследовании $G$-структур на многообразиях// Итоги науки и техн. Совр. мат. прилож. Темат. обз. - 2002. - 30. - С. 5-124.

13. Полякова К. В. Тангенциальнозначные формы 2-го порядка// Мат. заметки. -2019 . - 105, № 1 . C. $84-94$.

14. Розенфельд Б. А. Многомерные пространства. - М.: Наука, 1966.

15. Шевченко Ю. И. Оснащения центропроективных многообразий. - Калининград: КГУ, 2000.

16. Шевченко Ю. И. Аффинная, коаффинная и линейная факторгруппы в подгруппе проективной группы// в кн.: Проблемы мат. и физ. наук. - Калининград, 2002. - С. 38-39.

17. Шелехов A. М. О три-тканях, образованных семействами окружностей// Тр. Междунар. геом. центра. -2012 . - 5, № 2. - C. 6-16.

18. Akivis M. A., Goldberg V. V. A conformal differential invariant and the conformal rigidity of hypersurfaces// Proc. Am. Math. Soc. - 1997. - 125, № 8. - P. 2415-2424.

Белова Ольга Олеговна

Балтийский федеральный университет им. И. Канта, Калининград

E-mail: olgaobelova@mail.ru 